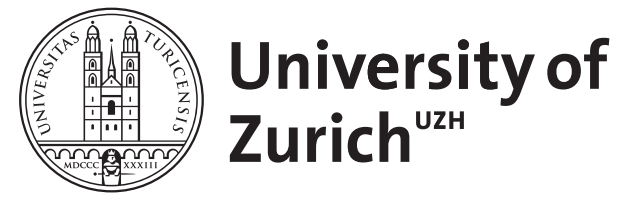

\title{
Sox5 and chromatophores: switching pigment cell fates
}

Debbache, Julien ; Sommer, Lukas

DOI: https://doi.org/10.1111/pcmr.12296

Posted at the Zurich Open Repository and Archive, University of Zurich ZORA URL: https://doi.org/10.5167/uzh-100802

Journal Article

Accepted Version

Originally published at:

Debbache, Julien; Sommer, Lukas (2014). Sox5 and chromatophores: switching pigment cell fates. Pigment Cell Melanoma Research, 27(6):1004-1005.

DOI: https://doi.org/10.1111/pcmr.12296 


\section{Sox5 and chromatophores: Switching pigment cell fates}

\section{Julien Debbache and Lukas Sommer}

e-mail: julien.debbache@uzh.ch and lukas.sommer@anatom.uzh.ch

Coverage on: Nagao Y, Suzuki T, Shimizu A, Kimura T, Seki R, et al. (2014) Sox5 Functions as a Fate Switch in Medaka Pigment Cell Development. PLoS Genet 10(4): e1004246.

doi:10.1371/journal.pgen.1004246

Unlike mammals and birds, certain fish species possess more than one type of neural crest-derived pigment cells. Zebrafish and medaka, among others, harbour chromatophores, which include black melanophores, iridescent iridophores and yellow xanthophores. Furthermore, the medaka bears a distinct chromophore population called leucophores. While the specification of melanophores and iridophores from a common progenitor has been linked to the expression of Mitf and FoxD3 respectively (Curran et al. 2009; Curran et al. 2010), the leucophores' origin and differentiation process remain poorly understood. Previous studies have suggested a close relationship between xanthophores and leucophores based on their shared biochemical properties, but never linked them developmentally (Braasch et al. 2007). Nagao et al., through the molecular characterization of several spontaneous mutants harbouring defects in xanthophore and leucophore numbers, shed light on the transcription factor Sox 5 as a critical developmental switch in their respective differentiation.

First, Nagao and colleagues determined that the $\mathrm{ml}-3$ locus encodes a factor switching specifically the fate of xanthophores in favour of leucophores in a dose-dependent fashion without altering melanocyte or iridophore populations. These data highlight the presence of a common progenitor cell for these two lineages. Using positional cloning, the authors then linked the $\mathrm{ml}-3$ locus to the transcription factor Sox5, a gene previously described to harbour the capacity to modulate the activity of several members of the SoxE transcription factor family, including Sox10, a critical player of neural crest and pigment cell development (Stolt et al. 2008; Harris et al. 2013). Nagao et al. identified the $\mathrm{ml}-3$ mutation as resulting from an aberrant splicing of exon7, which triggers a premature STOP codon and truncation of Sox5 prior to its HMG box and 2nd coiled-coil domain. Gene silencing using morpholinos and independent mutagenesis both led to increased leucophore numbers and absence of xanthophore without affecting other pigment cells, further validating Sox 5 loss of function as responsible for the $\mathrm{ml}-3$ phenotype.

The authors were then able to show, with the help of cell transplantation experiments between $\mathrm{ml}-3$ and control embryos and vice versa, that cells originating from Sox5-deficient embryos fail to give rise to any significant number of xanthophores. In contrast, control cells were able to generate normal numbers of xanthophores in $\mathrm{ml}-3$ mutant fishes. Furthermore, they demonstrated an aberrant increase in leucophore numbers in embryos lacking Sox5. However, the observed changes in pigment cell subpopulations, were not only due to cell-autonomous effects of sox 5 deficiency, but also caused by the lack of xanthophores allowing leucophores to ectopically populate chromatophore free territories. Hence, Nagao et al. show that Sox 5 acts as fate switch in pigment cell lineages, but the cell numbers constituting these lineages are apparently controlled independently from Sox 5.

Finally, a phenotypic comparison of If-2 (pax7a) mutants, which lack both xanthophores and leucophores (Kimura et al. 2014), with $\mathrm{ml}-3$ mutant embryos enabled the authors to identify a genetic cascade resulting in the specification of both of the above mentioned lineages. Using detailed embryonic in situ hybridization expression analysis, Nagao et al. described how Sox 5 expression in $\mathrm{ml}-3$ is 
maintained in the embryonic neural tube and in neural crest derivatives in the medial pathway, but not in areas associated with xanthophores, further underlining the lineage-specific Sox 5 dependency of xanthophores. Similar experiments in pax7a-deficient embryos revealed the same lack of Sox5expressing cells in the lateral trunk region. Together with the phenotypic data of $/ \mathrm{f}-2 / \mathrm{ml}-3$ double mutant showing no difference from the single If-2 mutant phenotype, the authors show that Pax7a is required for the generation of a common progenitor for xanthophores and leucophores and is genetically upstream of sox5. However, the Sox 5 expression status in this progenitor cell population is unclear. Indeed, the in situ hybridization analysis presented by the authors reveals a relatively broad expression of Sox 5 in pre-migratory neural crest cells. Thus, two scenarios are possible: a repression of Sox 5 upon leucophore differentiation from a Sox $5(+)$ progenitor population, where Sox 5 is not critical for the survival of the latter; or a de novo expression of Sox 5 upon xanthophore differentiation from a Sox5(-) progenitor population. In any case, the data indicate that Sox 5 expression levels must be tightly regulated during pigment cell development, although the factors acting upstream of Sox 5 have not yet been identified. The position of Sox 5 as critical switch between xanthophore and leucophore fates also suggests that Sox 5 gain of function would prevent the generation of leucophores to the benefit of xanthophore derivatives, but this remains to be demonstrated. Additionally, it would be of interest to address whether functional manipulation of Sox 5 or of a related factor would be sufficient to induce emergence of leucophores in Zebrafish, which normally lack this pigment cell lineage but have xanthophores.

In summary, Nagao et al. have identified Sox 5 as a fate switch in the differentiation process between the xanthophore and leucophore cell population from a Pax7a-dependent common progenitor, in Medaka. Although the authors propose an interaction model involving Sox10 and Pax7a and resulting in a modulation of their respective targets, the precise modus operandi of Sox 5 in the differentiation of xanthophores by target gene-specific regulation has yet to be characterized. Their data strengthen the biological importance of Sox 5 as a central genetic regulator, not only in fish, but perhaps also in mammalian pigment cells as previously suggested by Stolt et al. (Stolt et al. 2008). Moreover, this study provides another intriguing example of how differential expression of Sox proteins controls fate decision processes during neural crest development (John et al. 2011)

Braasch, I., Schartl, M. \& Volff, J.-N., 2007. Evolution of pigment synthesis pathways by gene and genome duplication in fish. BMC evolutionary biology, 7, p.74.

Curran, K. et al., 2010. Interplay between Foxd3 and Mitf regulates cell fate plasticity in the zebrafish neural crest. Developmental biology, 344(1), pp.107-18.

Curran, K., Raible, D.W. \& Lister, J. a, 2009. Foxd3 controls melanophore specification in the zebrafish neural crest by regulation of Mitf. Developmental biology, 332(2), pp.408-17.

Harris, M.L. et al., 2013. A dual role for SOX10 in the maintenance of the postnatal melanocyte lineage and the differentiation of melanocyte stem cell progenitors. PLoS genetics, 9(7), p.e1003644.

John, N. et al., 2011. Transforming growth factor $\beta$-mediated Sox10 suppression controls mesenchymal progenitor generation in neural crest stem cells. Stem cells (Dayton, Ohio), 29(4), pp.689-99. 
Kimura, T. et al., 2014. Leucophores are similar to xanthophores in their specification and differentiation processes in medaka. Proceedings of the National Academy of Sciences of the United States of America.

Stolt, C.C. et al., 2008. The transcription factor Sox 5 modulates Sox10 function during melanocyte development. Nucleic acids research, 36(17), pp.5427-40. 